\title{
Revision Strategies
}

\author{
Kristin Messuri PhD
}

\begin{abstract}
Revision is difficult for many writers. This article outlines a systematic approach to revision, including concrete strategies for addressing both higher-order concerns and lower-order concerns.
\end{abstract}

Key words: writing, medical writing, medical manuscript, periodicals as topic

Regardless of writers' levels of experience or areas of expertise, many struggle with revision, a component of the writing process that encompasses everything from transformative changes in content and argumentation to minor corrections in grammar and punctuation. Perhaps because revision involves so many forms of modification, it is the focus of most scientific writing guides and handbooks, including the articles in this series. ${ }^{1-4}$ Revision can be daunting; how does one progress from an initial draft (which is often called a "rough draft," with good reason) to a polished piece of scholarly writing?

This article offers several possible answers to this question. Developing a process for revision can help writers produce thoughtful, polished texts and grow their written communication skills. Therefore, this article offers you, as a writer, a systematic approach to revision, including strategies to employ at every step of the process.

Corresponding author: Kristin Messuri, Ph.D. Contact Information: kristin.messuri@gmail.com DOI: 10.12746/swrccc 2016.0414.190

\section{A System for Approaching Revision}

Generally, revision should be approached in a top-down manner by addressing higher-order concerns (HOCs) before moving on to lower-order concerns (LOCs). In writing studies, the term "higher order" is used to denote major or global issues such as thesis, argumentation, and organization, whereas "lower order" is used to denote minor or local issues such as grammar and mechanics. ${ }^{5}$ The more analytical work of revising HOCs often has ramifications for the entire piece. Perhaps in refining the argument, a writer will realize that the discussion section does not fully consider the study's implications. Or, a writer will try a new organizational scheme and find that a paragraph no longer fits and should be cut. Such revisions may have far-reaching implications for the text. Dedicating time to tweaking wording or correcting grammatical errors is unproductive if the sentence will be changed or deleted. Focusing on HOCs before LOCs allows writers to revise more effectively and efficiently.

\section{REVISION StRATEgIES}

Bearing in mind the general system of revising from HOCs to LOCs, you can employ several revision 
strategies. Begin by evaluating how your argument addresses your rhetorical situation-that is, the specific context surrounding your writing, including the audience, exigence, and constraints. ${ }^{6}$ For example, you may write an article describing a new treatment. If the target journal's audience comes from a variety of disciplines, you may need to include substantial background explanation, consider the implications for practitioners and scholars in multiple fields, and define technical terms. By contrast, if you are addressing a highly specialized audience, you may be able to dispense with many of the background explanations and definitions because of your shared knowledge base. You may consider the implications only for specialists, as they are your primary audience. Because this sort of revision affects the entire text, beginning by analyzing your rhetorical situation is effective.

After addressing the implications of your rhetorical situation, focus on other HOCs. Analyze your thesis or main argument for clarity. Compare your abstract to your entire text and see if any content is missing or, conversely, if any extraneous information is present. Then, evaluate the global organization of your text by writing a reverse outline. Unlike traditional outlines, which are written before drafting, reverse outlines reflect the content of written drafts. In a separate document or in your text's margins, record the main idea of each paragraph. Then, consider whether the order of your ideas is logical. This method also will help you identify ideas that are out of place or digressive. You may also evaluate organization by printing the text and cutting it up so that each paragraph appears on a separate piece of paper. You may then easily reorder the paragraphs to test different organizational schemes.

Next, revise for paragraph organization and coherence. You may begin by highlighting each topic in a different color. If topics are scattered throughout different paragraphs, you may need to reorganize to make paragraphs more coherent. Then, evaluate each paragraph's topic sentence (the first sentence of the paragraph, which usually transitions from the previous paragraph and/or identifies the current paragraph's main argument). You may need to rewrite or add topic sentences so that they guide the reader into each paragraph.

Once you have considered paragraphing, analyze your use of source material. Check any paraphrases and quotations against the original texts. Quotations should replicate the original author's words, while paraphrases should maintain the original author's meaning but have altered language and sentence structures. For each source, confirm that you have adhered to the preferred style guide for the target journal or other venue.

Finally, consider individual sentences in terms of grammar, mechanics, and punctuation. Many LOCs can be revised by isolating and examining different elements of the text. Read the text sentence by sentence, considering the grammar and sentence structure. Remember, a sentence may be grammatically correct and still confuse readers. ${ }^{2,7}$ If you notice a pattern-say, a tendency to misplace modifiers or add unnecessary commas-read the paper looking only for that error. Read the document backwards, word for word, looking for spelling errors. Throughout the writing process and especially at this stage of revision, keep a dictionary, a thesaurus, and a writing handbook nearby.

Strategies such as reading aloud and seeking feedback are useful at all points in the revision process. Reading aloud will give you distance from the text and prevent you from skimming over what is actually written on the page. This strategy will help you to identify both HOCs, such as missing concepts, and LOCs, such as typos. Additionally, seeking feedback will allow you to test your ideas and writing on real readers. Seek feedback from readers both inside and outside of your target audience in order to gain different perspectives.

\section{Conclusion: DeVeloping a PERSonal} WRITING PROCESS

The advice dispensed above is best understood as a set of best practices. Writing, including revising, is not a process that can be standardized; different practices will work better for different writers 
at different times and for different purposes and projects. In a sense, the best practice is the practice that works. As you try the revision strategies outlined in this article and create and explore your own, try to be mindful of which approaches work best for you. Consider ending each writing and revision session by reflecting on what worked well and what you would like to improve. Doing so will help you to develop a personal writing process that allows you to produce thoughtful, effective texts.

Author Affiliations: Kristin Messuri is the Associate Director of the University Writing Center at Texas Tech University / Texas Tech University Health Sciences Center in Lubbock, TX

Submitted: 3/29/2016

Published electronically: 4/15/2016

Conflict of Interest Disclosures: None

\section{REFERENCES}

1. Messuri K. Choosing and using verbs. Southwest Respir Crit Care Chron 2016; 4 (13): 57-59.

2. Messuri K. Clarity in medical writing. Southwest Respir Crit Care Chron 2015; 3 (12): 56-58.

3. Goodman NW, Edwards MB, Langdon-Neuner E. Medical writing: a prescription for clarity. 4th ed. Cambridge (GB): Cambridge University Press; 2014.

4. Matthews JR, Matthews RW. Successful scientific writing: a step-by-step guide for the biological and medical sciences. 4th ed. Cambridge (GB): Cambridge University Press; 2014.

5. McAndrew DA, Registad TJ. Tutoring writing: a practical guide for conferences. Portsmouth (NH): Boynton/Cook; 2001.

6. Bitzer L. The rhetorical situation. Philos Rhetoric 1968; 1 (1): 1-14.

7. Sheffield, N. Scientific Writing Resource [Internet]. Durham (NC): Duke University Graduate School; 2011-2013 [cited 2016 Mar 22]. Available from: https://cgi.duke.edu/web/sciwriting/index.php/. 